\title{
Lorentz Force and Joule Heat Induced in an Electrically Conducting Plate Moving With Time-Dependent Velocity Under the Influence of a Homogeneous Magnetic Field
}

\author{
Christian Weidermann ${ }^{1}$, Igor Sokolov ${ }^{1}$, and Andre Thess ${ }^{2}$ \\ ${ }^{1}$ Institute of Thermodynamics and Fluid Mechanics, Ilmenau University of Technology, Ilmenau 98684, Germany \\ ${ }^{2}$ German Aerospace Center Institute of Engineering Thermodynamics, Pfaffenwaldring 38-40, \\ 70569 Stuttgart, Germany
}

\begin{abstract}
This paper investigates the interaction of an initially uniform magnetic field with an electrically conducting slab that moves perpendicularly to the magnetic field with arbitrary time-dependent velocity. It is demonstrated that the problem of determining the time-dependent Lorentz force and the time-dependent Joule heat in the slab is mathematically equivalent to solving a 1-D heat diffusion problem with time-dependent boundary conditions and to submitting the solution to a nonstandard postprocessing procedure. For the particular case of an impulsively driven slab we exploit the mathematical analogy between magnetic diffusion and heat diffusion by translating a textbook solution of the corresponding heat-transfer problem into exact and previously unknown relations for Lorentz force and Joule heat. Moreover, we use a 1-D finite-difference code to investigate more general time dependencies of the velocity including smooth accelerations and random velocity changes. Our numerical determination of reaction times $\left(T_{98}\right)$ of the Lorentz force in the case of smooth accelerations provides a useful design tool for the development of Lorentz force flowmeters with short reaction times.
\end{abstract}

Index Terms - Flow measurement, flowmeters, induction heating, liquid metals, magnetohydrodynamics, metallurgy.

\section{INTRODUCTION}

W HEN an electrically conducting material moves across magnetic field lines, eddy currents are induced in the conductor. These currents carry an additional magnetic field which leads to a deformation of the applied magnetic field lines [1], [2]. Thus, the conducting material experiences a braking Lorentz force. Moreover, the eddy currents generate Joule heat which leads to an increase of the temperature of the material [3]-[7]. The object of this paper is to formulate and solve a model which predicts the time dependence of the Lorentz force and Joule heat for a simple geometry of the conductor and a homogeneous magnetic field. Our investigation is motivated by two applications sketched in Fig. 1.

Regarding the motion of the electrical conductor we distinguish translational motion [Fig. 1(a)] with velocity $V$ and rotational motion [Fig. 1(b)] with angular velocity $\omega$. The first case describes the basic principle of a noncontacting flow measurement method called Lorentz force velocimetry (LFV). It has been previously studied by Thess et al. [8], [9]. This method uses the Lorentz force to determine the mean velocity of the fluid flow in a closed pipe or an open channel. With the velocity of the fluid flow it is possible to calculate the physical quantities mass flux $\dot{m}$, volume flux $\dot{V}$ or, the within an arbitrary time interval transported mass $M$. The measurement devices that use Lorentz force velocimetry are called LoFoMeter. These devices are used for automation purposes in various industrial applications and measure the time-dependent mass flux $\dot{m}(t)$. Particularly for feedback control loops the

Manuscript received September 5, 2013; revised November 5, 2013, January 14, 2014, and February 25, 2014; accepted March 2, 2014. Date of publication March 5, 2014; date of current version August 15, 2014 Corresponding author: C. Weidermann (e-mail: christian.weidermann@tuilmenau.de).

Digital Object Identifier 10.1109/TMAG.2014.2309938

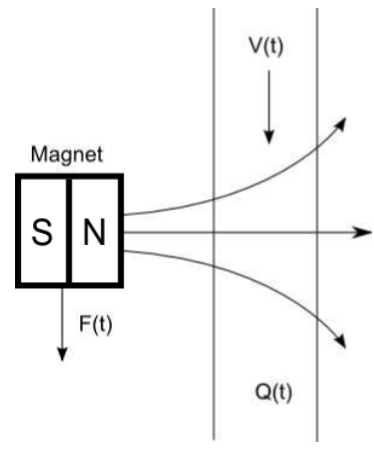

(a)

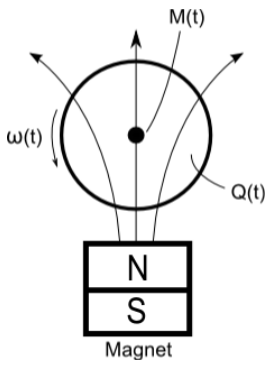

(b)
Fig. 1. Basic principle of (a) LFV and of (b) dc induction heating for a rotating cylinder. In LFV the time-dependent Lorentz force $F(t)$ is measured to deduce the time-depending flow velocity $V(t)$. In dc induction heating either a time varying magnetic field or the motion of an electrically conducting, nonmagnetic ingot in a static magnetic field leads to the generation of Joule heat $Q(t)$ in the material and heats it up to a certain temperature for material treatment like hardening.

dynamics of the measurement device are directly linked to the performance of the system. In case of a sudden jump in the mass flux, the LoFometer cannot follow the input exactly. The measurement signal of the LoFometer is delayed with a characteristic reaction time $T_{\xi 98}$. This quantity states the required time that is needed by the measurement device to reach $98 \%$ the new value. The reaction time shows the dynamical limits of the device. Therefore, it is significant to investigate the time response of the Lorentz force and to provide a simple mathematical model. The second case of rotational motion is related to direct current (dc)-induction heating. The process of induction heating is well known from [10]. A nonmagnetic material can be heated up either by a time-depending sinusoidal magnetic field [alternating current (ac)-induction heating] or by motion within a static 
magnetic field (dc-induction heating). Mach et al. [11] and Fabbri et al. [12] studied the induction heating of cylindrical nonmagnetic ingots. But there is no detailed model about the time-dependent Joule heat. With a sudden jump in the rotational velocity of the billet, the Joule heat does not follow the velocity change exactly. There is delay with a characteristic reaction time $T_{\psi} 98$ comparable with the Lorentz force. A better understanding of the transient process helps to optimize the startup curves in industrial processes. The transient behavior of LFV and dc induction heating is mathematically nontrivial. In general, the interaction between magnetic fields and electrically conducting materials is bidirectional and fully coupled. A change in the velocity of the moving conductor takes influence on the shape of the induced magnetic field and the magnetic field itself exerts a braking force on the moving conductor. The intensity of the coupling and the interaction between both is expressed by a nondimensional parameter called the magnetic Reynolds number $R m$. The magnetic Reynolds Number is defined as ratio of convection of magnetic field to diffusion of magnetic field. For small values of $R m \ll 1$ magnetic diffusion is dominant. There is almost no deformation of the magnetic field. For large values of $R m \gg 1$ convection of magnetic field is dominant and magnetic diffusion is weak for instance the earth magnetic field exposed to a solar storm [3]-[7]. Parker [13] intensively studied the effect of reconnexion of lines of magnetic force in 1966 for a rotating cylinder in an initially uniform magnetic field. He found an analytical solution for a simplified mathematical model and has shown that lines of magnetic force reconnect to form closed loops during the transient phase. Perry and Jones [14] investigated the induction of eddy currents in a solid conducting cylinder. All studies are primarily concerned with deformation of the magnetic field owing to the rotational movement of a cylindrical conductor. But there are only weak contributions regarding the transient of Lorentz force and Joule heat. After intensive literature research there is no scientific contribution that investigates the time dependence of the Lorentz force and the Joule heat, when a moving metal sheet interacts with a uniform magnetic field. It appears that the effect has not been sufficiently studied. The aim of this paper is the investigation of the transient phase of the Lorentz force and Joule heat for different motion setups of the moving conductor. Therefore, we obtain analytical solutions for three motions setups, namely step-function, exponential function, and sinusoidal motion of the plate. Later on we apply a finite difference code to achieve solutions for sophisticated motion setups expected in real applications. The outcome is a characteristic nondimensional time $T_{98}$, which means that the transient phase is completed to $98 \%$. This value is a significant indicator for the dynamics of the flow measurement method of LFV. This paper is mainly focused on academic purposes. However, there is a practical background in the subject of flow measurement. Today LFV is established as a universal noncontact flow measurement method for liquids like metal melts or electrolytes [15]-[17]. Here the time-resolved measurement of the mean velocity of the fluid flow requires information about the dynamics Lorentz force velocimetry. This paper theoretically investigates this subject for the first time. In Section II, a mathematical model for the time response of a canonical problem is developed and analytical solutions for different motion setups are obtained. In Section III, a finite difference code is used to calculate sophisticated motion setups of the metal sheet. In Section IV, we summarize the results and give an outlook for upcoming investigations. As an important remark, we would like to point out that we do not consider the self-magnetic pinch-effect. Since we deal with solid metals, the effect has no influence on a conductor structure and therefore was excluded from our consideration. An interested reader can refer to [18] or [19] in order to have a deeper look at this phenomenon.

\section{MATHEMATICAL MODEL}

Here we consider three cases of time-dependent velocity. First, a stepwise velocity is taken as a basic one. Then, the analytical solution for the velocity signal with final acceleration is shown. Finally, we discuss a velocity input, which changes periodically over time.

\section{A. Case 1: Stepwise Velocity Signal}

In general, the interaction of a magnetic field $\vec{B}$ with an electrically conducting material that moves with a velocity $\vec{V}$ is described by the magnetic field transport equation [3], [4], [7], [20], which has the general form

$$
\frac{\partial \vec{B}}{\partial t}=\nabla \times(\vec{V} \times \vec{B})+\lambda \nabla^{2} \vec{B}
$$

Here $\lambda=1 / \mu \sigma$ stands for the magnetic diffusivity, where $\mu=4 \pi \times 10^{-7} H \cdot m^{-1}$ is the magnetic permeability and $\sigma$ is the electrical conductivity of a material. A full numerical analysis of the problems is shown in Fig. 1 to determine $F(t)$ in $\left[N / m^{2}\right]$ and $Q(t)$ in $\left[W / m^{2}\right]$ for a given $V(t)$ in case of Lorentz force velocimetry or $M(t)$ and $Q(t)$ for a given $\omega(t)$ in case of dc induction heating requires numerical solutions of the 3-D equation (1) and is beyond the scope of this paper. Here we study a simple model that is amenable to either analytical or inexpensive 1-D numerical treatment and can therefore provide better insight into the time dependence of the Lorentz force and Joule heat than a full 3-D simulation.

Our model is shown in Fig. 2. We assume a laterally infinite plate with thickness $D$. The plate is electrically conducting and its electrical conductivity is $\sigma$. An externally applied uniform magnetic field $B_{0}$ penetrates the plate. For $t<0$ the plate is at rest and at time $t=0$ the plate starts moving with a time-dependent velocity $V(t)$. First of all we will consider the case when the velocity is constant and equals to $V_{0}$ for $t>0$. Then we will investigate the more general case when the plate is smoothly accelerated and reaches its asymptotic (constant) velocity $V_{0}$ for $t \rightarrow \infty$. In this case we will use this asymptotic value $V_{0}$ as the velocity scale. Finally, we will discuss the problem, where the velocity changes periodically. Before coming further, we would like to switch to nondimensional parameters. Velocity of the plate is nondimensionalized by $V_{0}$, the space coordinate is referred to the thickness of the plate $D$ and extends for symmetry reasons from $z=0$ to $z=1$. The time coordinate $t$ is referred to the magnetic diffusion time $\tau=\mu \sigma D^{2}$ as a characteristic time scale and $R m B_{0}$ 


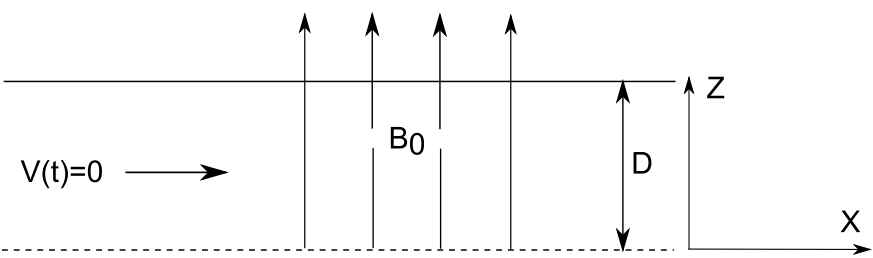

(a)

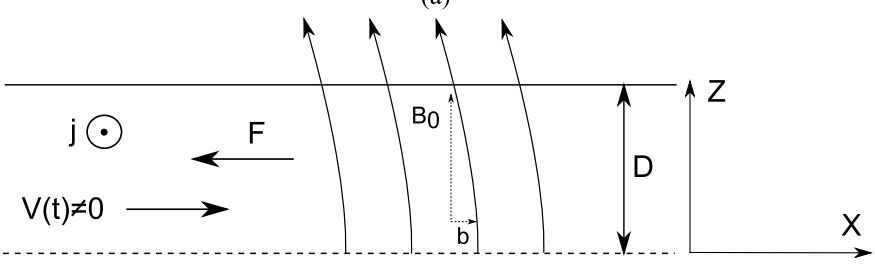

(b)

Fig. 2. Definition of the model. (a) Interaction of a laterally unbounded electrically conducting plate with a homogeneous magnetic field $B_{0}$. Because of the symmetry, only a half of the plane is considered. If the plate experiences no motion, the applied magnetic field $B_{0}$ is not distorted. (b) In case of nonzero velocity, eddy currents $j$ ensue that leads to a primary magnetic field disturbance by an induced magnetic field $b$. An interaction between the induced magnetic field and eddy currents gives rise to the Lorenz force $F$, which opposes the flow.

is used to nondimensionalize the induced magnetic field $b$, where $R m=\mu \sigma V D$ is magnetic Reynolds number [3]. A complete list of reference parameters and the derivation of the second boundary condition are shown in Appendix A. To determine the Lorentz force and the Joule heat, we need to compute the distribution of the magnetic field inside the plate. To accomplish this task, we start with the observation that owing to the motion of the plate a secondary magnetic field $\vec{b}$ is induced. This field is proportional to $R m B_{0}$ [3] and perturbs the primary (applied) magnetic field. For our simple geometry it can be shown that $\vec{b}$ has only one nonzero component and depends only on $z$ and on $t$. Hence we can write the velocity field and magnetic field as following [21]:

$$
\begin{aligned}
\vec{V} & =V(t) \vec{e}_{x} \\
\vec{B} & =\vec{e}_{z}+b(z, t) \vec{e}_{x} .
\end{aligned}
$$

Taking these equations, inserting them into (1) and observing that outside the plate the secondary magnetic field is independent of $z$, it can be readily demonstrated that the distribution of the secondary magnetic field $b(z, t)$ in the moving plate is described by the 1-D diffusion equation

$$
\frac{\partial b}{\partial t}=\frac{\partial^{2} b}{\partial z^{2}}
$$

with the initial condition $b(z, 0)=0$ and with the mixed boundary conditions [21]

$$
\begin{aligned}
\left.b(z, t)\right|_{z=0} & =0 \\
\left.\frac{\partial b(z, t)}{\partial z}\right|_{z=1} & =-V(t) .
\end{aligned}
$$

The first boundary condition involves the fact that the solution of the magnetic field is expected to be antisymmetric. The second boundary condition is a kind of matching condition and can be obtained by integrating (1) over an $\epsilon$-neighborhood of the boundary. Notice that this problem is mathematically equivalent to the heat-transfer problem in a plate which is cooled down from above with time-dependent cooling rate $-V(t)$ and having constant zero temperature on the other side. This analogy is neither new nor surprising, it is indeed well known in magnetohydrodynamics [3]. The nontrivial mathematical aspect of the present problem is in the fact that the determination of the Lorentz force and the Joule heat require the computation of nonstandard quantities that are unknown in heat-transfer problems. We thereby demonstrate that a wide variety of exact analytical solutions of 1-D heattransfer problems known from ([22] and references therein) can be easily converted to relations useful for the Lorentz force velocimetry and dc induction heating.

Once the magnetic field $b(z, t)$ has been determined, the nondimensional $y$-component of the electric current density is given by $j_{y}(z, t)=-\partial b / \partial z$. From these two quantities, the $x$-component of the Lorentz force density can be derived as $f(z, t)=j(z, t) b(z, t)$ and the volumetric generation of Joule heat is $q(z, t)=j^{2}(z, t)$. By integrating these two quantities over the thickness of the plate, we obtain the nondimensional time-dependent total Lorentz force $\xi(t)$ and the total Joule heat $\psi(t)$ as

$$
\begin{aligned}
\xi(t) & =b(1, t) \\
\psi(t) & =\int_{0}^{1} j^{2}(z, t) d z .
\end{aligned}
$$

Before proceeding to the discussion of the results, we shall write down the relations that are necessary to convert the nondimensional Lorentz force $\xi(t)$ and Joule heat $\psi(t)$ back into dimensional quantities that are necessary for practical purposes. The time-dependent Lorentz force per unit area (with unit Newton per square meter) is obtained from $\xi$ as

$$
F=-\sigma D v_{0} B_{0}^{2} \xi\left(\lambda t / D^{2}\right)
$$

where the time in $\xi$ should be taken in seconds. The timedependent Joule heat per unit area (with unit watt per square meter) is obtained from $\psi$ as

$$
Q=\sigma^{2} D v_{0}^{2} B_{0}^{2} \psi\left(\lambda t / D^{2}\right) .
$$

The rest of the paper is devoted to the derivation and discussion of $\xi(t)$ and $\psi(t)$ for different input signals $V(t)$. We start our discussion with the analytically solvable problem of a sudden jump in the velocity of the plate described by

$$
V(t)= \begin{cases}0, & \text { for } t \leq 0 \\ 1, & \text { for } t>0\end{cases}
$$

The solution of the corresponding heat-transfer problem is known from [23]. The magnetic field and eddy current distributions of this solution are [21]

$$
\begin{aligned}
& b(z, t)=\left[-z+\frac{8}{\pi^{2}} \sum_{n=0}^{\infty} \frac{(-1)^{n}}{(2 n+1)^{2}} \sin \left(\beta_{n} z\right) e^{-\alpha_{n} t}\right] \\
& j(z, t)=\left[1-\frac{4}{\pi} \sum_{n=0}^{\infty} \frac{(-1)^{n}}{(2 n+1)} \cos \left(\beta_{n} z\right) e^{-\alpha_{n} t}\right]
\end{aligned}
$$

where $\alpha_{n}=\left((2 n+1)^{2} / 4\right) \pi^{2}$ and $\beta_{n}=(2 n+1 / 2) \pi$.

Fig. 3(a) and (b) shows the magnetic flux density and the eddy currents as a function of $z$ for different instants of time. 


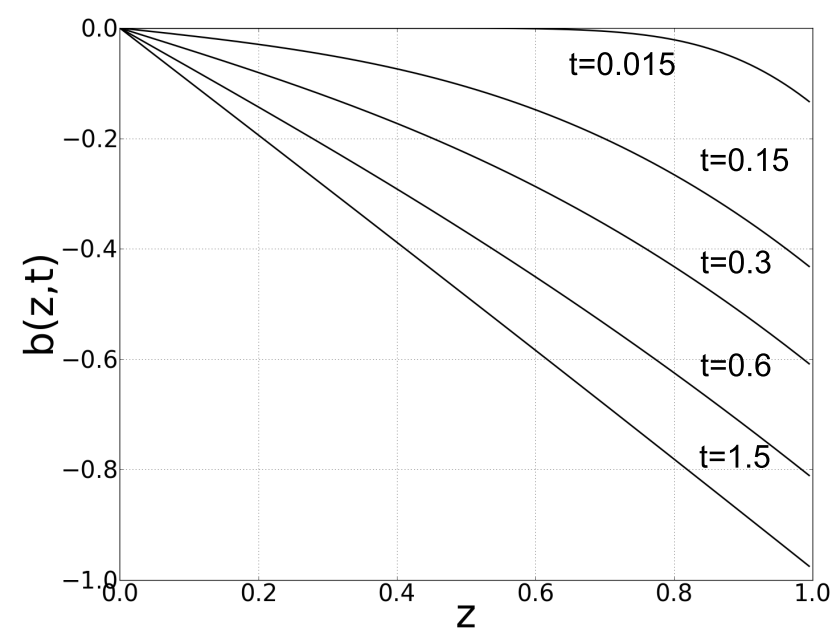

(a)

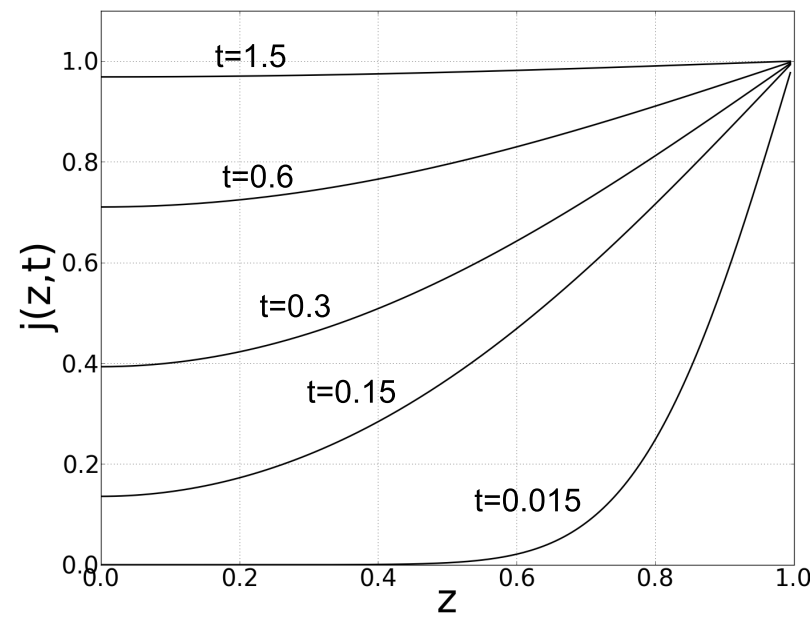

(b)

Fig. 3. Analytical solution of (a) magnetic flux density and (b) eddy current density for several instants of time as computed from (12) and (13).

Although the solution itself is not new, its application to the problem of magnetic diffusion leads to new conclusions. The flux density is antisymmetric with respect to $z=0$ whereas the eddy currents are symmetric. Both flux density and eddy currents are zero at $t=0$. When the plate starts moving, the secondary magnetic field continuously increases during transient phase. Later, the distribution of $b$ tends to a linear dependence on $z$ that corresponds to the steady-state heat transfer from the heated top to the cooled bottom of the plate in the corresponding heat-transfer problem. After a time of approximately $t=1.5$ the transient phase is completed (Fig. 3) and the magnetic field distribution and the eddy current distribution remain at a steady state. The eddy currents, which flow to oppose the flux change, reach a constant value after the transient phase and remain constant at steady state while the plate is moving with a constant velocity. Using (12) and (13) we compute our desired analytical expressions for the time-dependent Lorentz force $\xi(t)$ and Joule heat $\psi(t)$ as

$$
\begin{aligned}
\xi(t) & =\left[-1+2 \sum_{n=0}^{\infty} \frac{1}{\alpha_{n}} e^{-\alpha_{n} t}\right] \\
\psi(t) & =\left[1-4 \sum_{n=0}^{\infty} \frac{1}{\alpha_{n}} e^{-\alpha_{n} t}\left(1-\frac{1}{2} e^{-\alpha_{n} t}\right)\right]
\end{aligned}
$$

(see [21]). These quantities are shown in Fig. 4. Both are zero as long as the plate is at rest. After the jump in the velocity of the conductor the Lorentz force and the Joule heat increase and converge to their asymptotic values $\xi=\psi=1$ as $t \rightarrow \infty$. It is interesting that the Joule heat grows slower than the Lorentz force which is a consequence of the fact that the Joule heat is a quadratic function of $\partial b / \partial z$ whereas the Lorentz force depends linearly on $b$. Notice that due to the diffusive character of the magnetic field the dissipated power $Q$ is not equal to the instantaneous value of $F \cdot V$ as would be intuitively expected on the basis of a quasi-static picture.

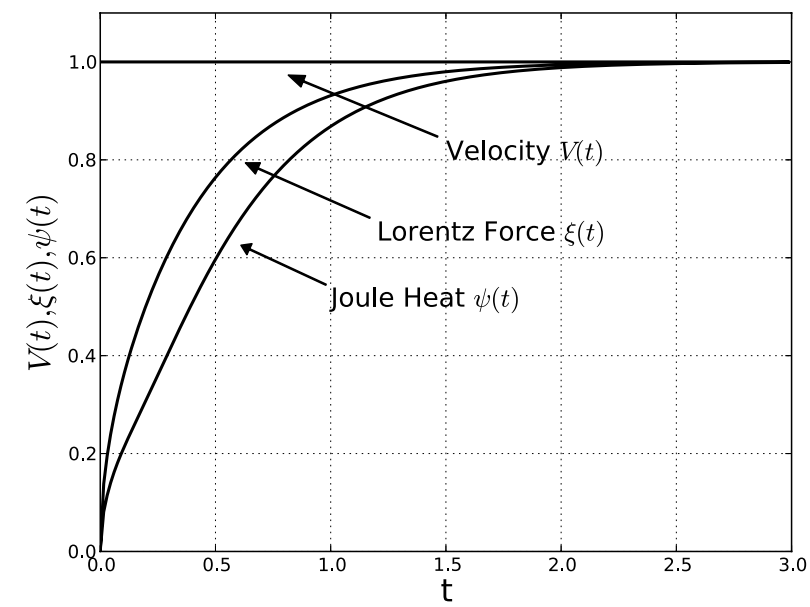

Fig. 4. Analytical solution of the Lorentz force absolute value $|\xi(t)|$ and Joule heat $\psi(t)$ in case of stepwise velocity signal. Both functions approach unity as $t \rightarrow \infty$.

By comparing (14) and (15) one can infer that the space harmonic damps at different rates. For higher value of $n$ the harmonics damp faster. This fact is reasonable, because it is sufficient to calculate only three terms in the series. For dimensional time $t>\tau=\mu \sigma D^{2}$ it is sufficient to calculate only the first term. Hence the fundamental time constant $\tau$, which is the longest time constant of the series, is the controlling time constant in the diffusion process. The functions $\xi(t)$ and $\psi(t)$ allow us to determine the reaction times $T_{98 \xi}$ and $T_{98 \psi}$, which are defined via $\xi\left(T_{98 \xi}\right)=0.98$ and $\psi\left(T_{98 \psi}\right)=0.98$, that is, when the Lorentz force and the Joule heat have reached $98 \%$ of their asymptotic values. Our solution yields the nondimensional reaction times $T_{98 \xi}=$ 1.48 and $T_{98 \psi}=1.76$ which for practical purposes can be translated into dimensional reaction times by multiplying then by a factor $\mu \sigma D^{2}$. As described in [17] and [21], $T_{98 \xi}$ can be directly used in the design of Lorentz force flowmeters for metallurgical applications where short reaction times are crucial. 
TABLE I

Reaction Time for Several $R m_{\tau}$ OF a Motion Setup Using Finite ACCELERATIONS EXPRESSED WITH AN EXPONENTIAL FUNCTION

\begin{tabular}{ccccc}
\hline$R m_{\tau}$ & $T_{98 v}$ & $T_{98 \xi}$ & $T_{98 \psi}$ & $T_{98 v} / T_{98 \xi}$ \\
\hline 1 & 3.91 & 4.34 & 5.03 & 0.9 \\
2 & 1.96 & 2.55 & 2.92 & 0.77 \\
3 & 1.30 & 2.05 & 2.35 & 0.63 \\
4 & 0.98 & 1.85 & 2.13 & 0.53 \\
5 & 0.78 & 1.74 & 2.02 & 0.45 \\
$\infty$ & 0 & 1.48 & 1.76 & 0 \\
\hline
\end{tabular}

\section{B. Case 2: Exponential Velocity Signal}

Practically stepwise velocity change can never be seen in a real experiment because it is impossible to change a conductor velocity with an infinite acceleration. Therefore, it is essential to investigate a problem where velocity is changed smoothly. Here we consider a second case, which is characterized by $V(t)=\left(1-e^{-R m_{\tau} t}\right)$, where $R m_{\tau}=\mu \sigma D^{2} / \tau$ is magnetic Reynolds number based on the time $\tau$ during which the velocity is changed. To obtain an analytical solution for that case, we refer to Duhamel's principle [24]. It says that if $P(t)$ is a time-varying boundary condition, then this value can be considered to be composed of an infinite number of infinitesimal step functions that switch on at different times. Thereby we can construct a system response to an input which varies continuously in time by adding the responses to each of these step units. If $b^{(0)}(z, t)$ is a response of a system to the unit step, a corresponding solution for nonstationary problem would be

$$
b(z, t)=\int_{0}^{t} P(\tau) \frac{\partial b^{(0)}(z, t-\tau)}{\partial t} d \tau .
$$

As the function $b^{(0)}(z, t)$ has been found in Case 1, we are able to find an exact solution for any velocity signal, which can be analytically integrated. Applying Duhamel's principle to the case 2 we obtain

$$
\begin{aligned}
b(z, t) & =2 \sum_{n=0}^{\infty}(-1)^{n} \sin \left(\beta_{n} z\right) \gamma\left(t, R m_{\tau}, \alpha_{n}\right) \\
j(z, t) & =2 \sum_{n=0}^{\infty}(-1)^{n+1} \beta_{n} \cos \left(\beta_{n} z\right) \gamma\left(t, R m_{\tau}, \alpha_{n}\right) \\
\xi(t) & =2 \sum_{n=0}^{\infty} \gamma\left(t, R m_{\tau}, \alpha_{n}\right)
\end{aligned}
$$

where

$$
\gamma\left(t, R m_{\tau}, \alpha_{n}\right)=\exp \left[\frac{1-e^{-\alpha_{n} t}}{\alpha_{n}}+\frac{e^{-R m_{\tau} t}-e^{-\alpha_{n} t}}{R m_{\tau}-\alpha_{n}}\right] .
$$

The response of the Lorentz force and the Joule heat for finite acceleration is shown in Fig. 5. A general shape of velocity resembles one in the first case, but here a function growth is slower with $T_{98 \xi}=1.88$. See Table I for the detailed response characteristics.

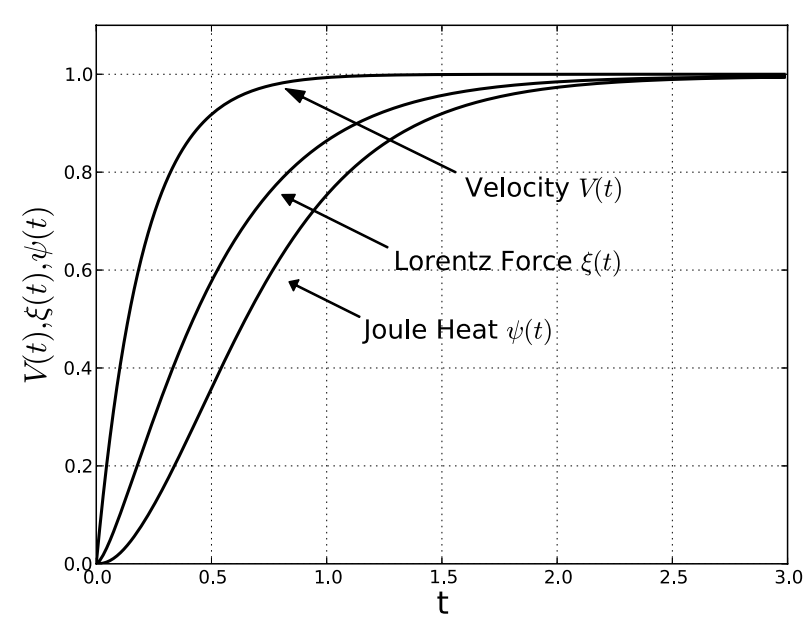

(a)

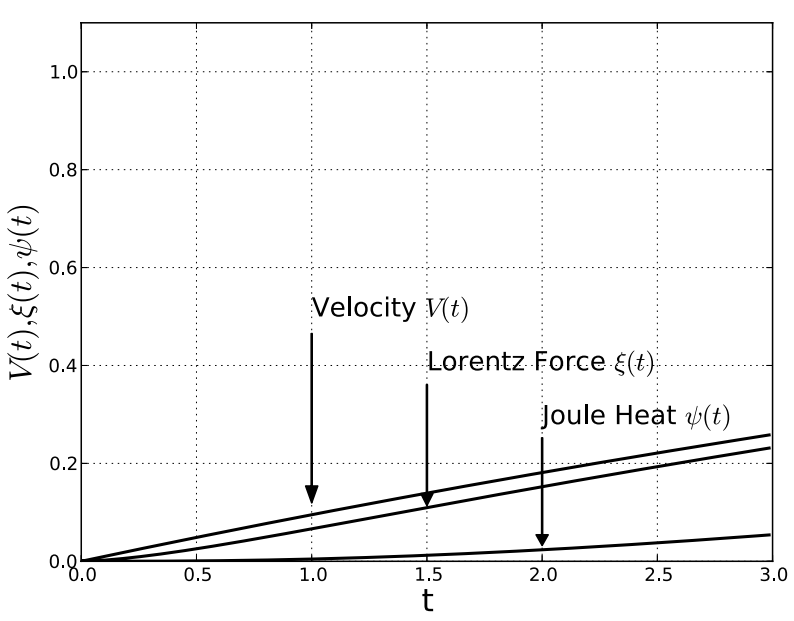

(b)

Fig. 5. Analytical solution of Lorentz force $\xi(t)$ and Joule heat $\psi(t)$ in case of $V(t)=1-\exp \left(R m_{\tau} t\right)$, where (a) $R m_{\tau}=5$ and (b) $R m_{\tau}=0.1$. Since the velocity increases more slowly than in the case with stepwise velocity signal, $T_{98} \xi$ is bigger than 1.48 at any $R m_{\tau}$.

\section{Case 3: Periodical Velocity Signal}

The case of sinusoidal motion is of a great importance, because it reveals a process of the magnetic field penetration into a material. In our third case, we assume the time-dependent velocity satisfies $V(t)=\cos \left(R m_{\omega} t\right)$, where $R m_{\omega}=\mu \sigma D^{2} \omega$ is magnetic Reynolds number based on the plate oscillation frequency. Using Duhamel's principle, the result for the magnetic flux density, eddy currents density, the Lorentz force, and Joule heat is

$$
\begin{aligned}
b(z, t) & =2 \sum_{n=0}^{\infty}(-1)^{n+1} \sin \left(\beta_{n} z\right) \varepsilon\left(t, R m_{\omega}, \alpha_{n}\right) \\
j(z, t) & =\pi \sum_{n=0}^{\infty}(-1)^{n}(2 n+1) \cos \left(\beta_{n} z\right) \varepsilon\left(t, R m_{\omega}, \alpha_{n}\right) \\
\xi(t) & =-2 \sum_{n=0}^{\infty} \varepsilon\left(t, R m_{\omega}, \alpha_{n}\right)
\end{aligned}
$$




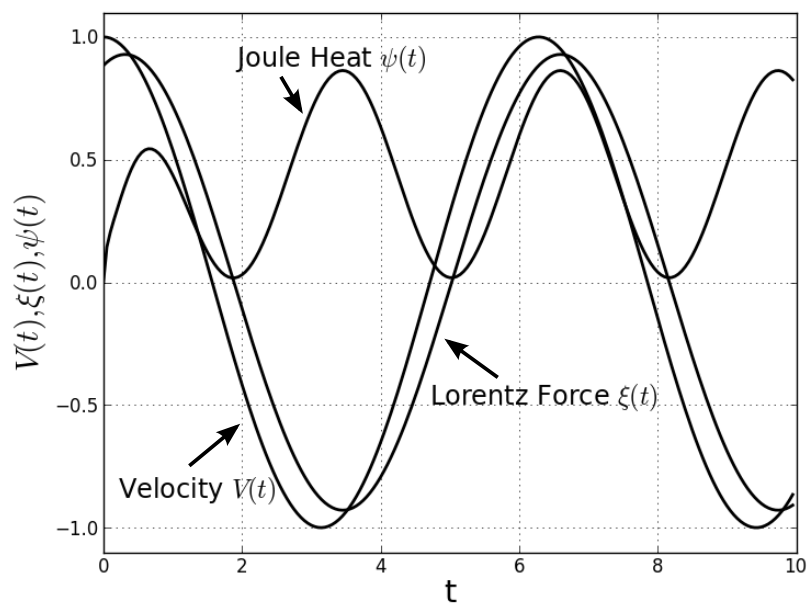

Fig. 6. Analytical solution of Lorentz force $\xi(t)$ and Joule heat $\psi(t)$ in case of $V(t)=\cos \left(R m_{\omega} t\right)$, where $R m_{\omega}=1$. There is a certain phase shift between $V(t)$ and $\xi(t)$, which tends to $\pi / 2$ when $R m_{\omega} \rightarrow \infty$.

$$
\psi(t)=\frac{1}{2} \pi^{2} \sum_{n=0}^{\infty}(2 n+1)^{2} \varepsilon^{2}\left(t, R m_{\omega}, \alpha_{n}\right)
$$

where

$$
\varepsilon\left(t, R m_{\omega}, \alpha_{n}\right)=\frac{R m_{\omega} \sin \left(R m_{\omega} t\right)+\alpha_{n} \cos \left(R m_{\omega} t\right)}{R m_{\omega}^{2}+\alpha_{n}^{2}} .
$$

Here we neglected an influence of the initial conditions (additional term $\left(-\alpha_{n} e^{-\alpha_{n} t}\right) /\left(R m_{\omega}^{2}+\alpha_{n}^{2}\right)$ in the function $\varepsilon$ ), because an asymptotic solution is of our primary interest. Here we neglect the term shown in Fig. 6 that the Lorentz force and Joule heat as a function of time. There are some remarkable facts. The first one is a doubled frequency of the Joule heat. This is due to the quadratic dependence on eddy current density and subsequently on the square of the velocity signal. The second one is that there is a phase shift $\Delta \phi_{\xi}$ between the velocity of the plate and the Lorentz force $\xi(t)$ as well as the phase shift $\Delta \phi_{\psi}$ between velocity signal and Joule heat $\psi(t)$. Moreover, the amplitudes of the Lorentz force $A_{\xi}$ and the Joule heat $A_{\psi}$ must depend on the frequency of the moving plate. Therefore, equations (22) and (23) can be expressed in a more descriptive way

$$
\begin{aligned}
\xi(t) & =A_{\xi}\left(R m_{\omega}\right) \cdot \cos \left(R m_{\omega} t+\Delta \phi_{\xi}\right) \\
\psi(t) & =A_{\psi}\left(R m_{\omega}\right) \cdot \cos \left(R m_{\omega} t+\Delta \phi_{\psi}\right) .
\end{aligned}
$$

With (22), one can analytically obtain an expression for the phase shift $\Delta \phi_{\xi}$

$$
\Delta \phi_{\xi}(t \rightarrow \infty)=\arctan \frac{\sum_{n=0}^{\infty} \frac{R m_{\omega}}{R m_{\omega}^{2}+\alpha_{n}^{2}}}{\sum_{n=0}^{\infty} \frac{\alpha_{n}}{R m_{\omega}^{2}+\alpha_{n}^{2}}} .
$$

Then it can be shown that

$$
\lim _{R m_{\omega} \rightarrow 0} \Delta \phi_{\xi}=0 \quad \lim _{R m_{\omega} \rightarrow \infty} \Delta \phi_{\xi}=\frac{\pi}{2} .
$$

It stands for the fact that the phase shift vanishes if $R m_{\omega} \rightarrow 0$ and the force exactly follows the velocity signal. On the other hand, if $R m_{\omega} \rightarrow \infty$ the phase shift $\Delta \phi \rightarrow \pi / 2$ while the

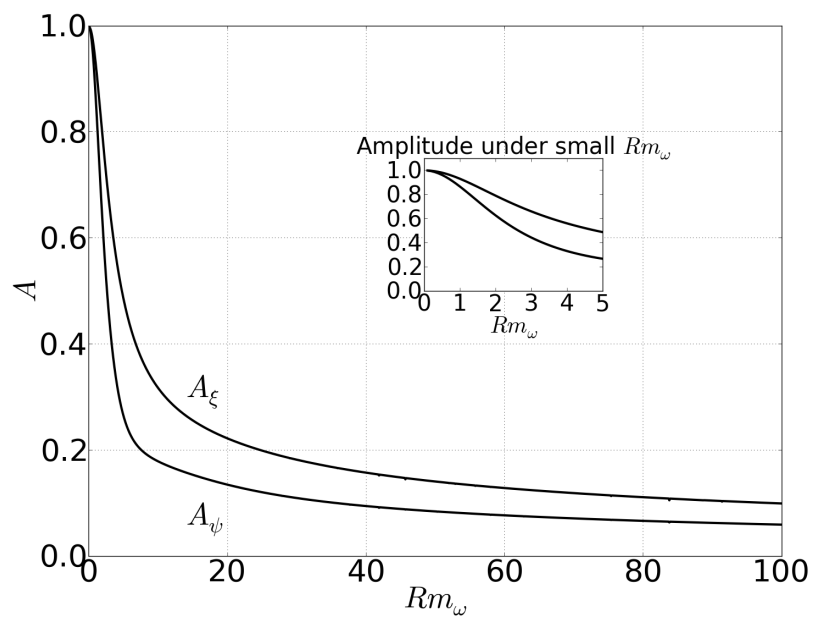

(a)

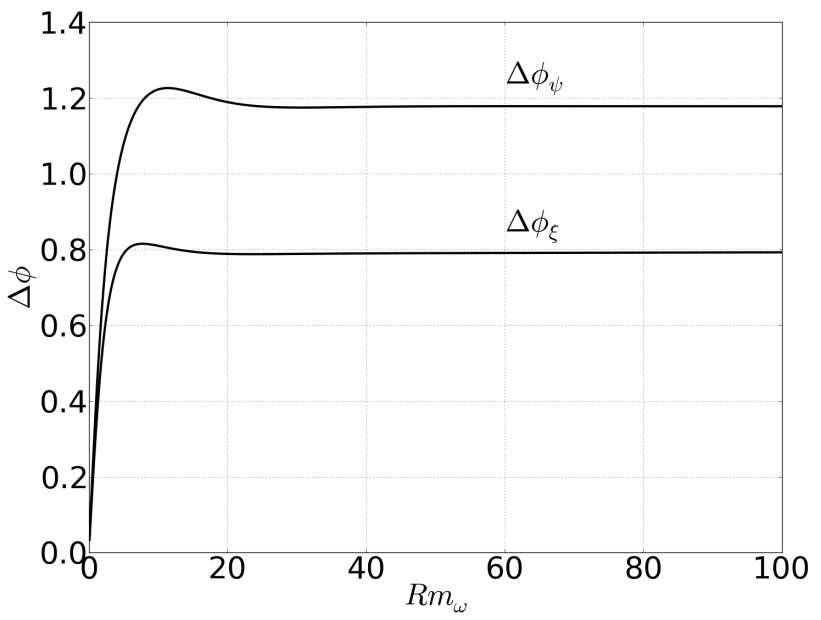

(b)

Fig. 7. (a) Amplitudes $A_{\xi}$ and $A_{\psi}$ and (b) phase shifts $\Delta \phi_{\xi}$ and $\Delta \phi_{\psi}$ as a function of $R m_{\omega}$. The behavior of these curves agrees well with an assumption that under high $R m_{\omega}$ magnetic field lines become frozen into a conductor and there is no relative movement anymore that leads to the absence of the Lorentz force.

Lorentz force amplitude tends to zero because of the skin effect [3]. Therefore, the amplitude $A_{\xi}$ decays approximately as $1 / R m_{\omega}$ and reads

$$
A_{\xi}=-2 \sum_{n=0}^{\infty} \frac{1}{\sqrt{1+\Psi^{2}}} \frac{\Psi R m_{\omega}+\alpha_{n}}{R m_{\omega}^{2}+\alpha_{n}^{2}}
$$

where

$$
\Psi=\frac{\sum_{n=0}^{\infty} \frac{R m_{\omega}}{R m_{\omega}^{2}+\alpha_{n}^{2}}}{\sum_{n=0}^{\infty} \frac{\alpha_{n}}{R m_{\omega}^{2}+\alpha_{n}^{2}}} .
$$

The same tendencies can be observed with the function $\psi(t)$. This is shown in Fig. 7. The absence of the phase shift between the velocity signal and the force response in case of small $R m_{\omega}$ is clear. As the oscillation frequency is small, the magnetic field has enough time to diffuse before being advected that allows the force to react 


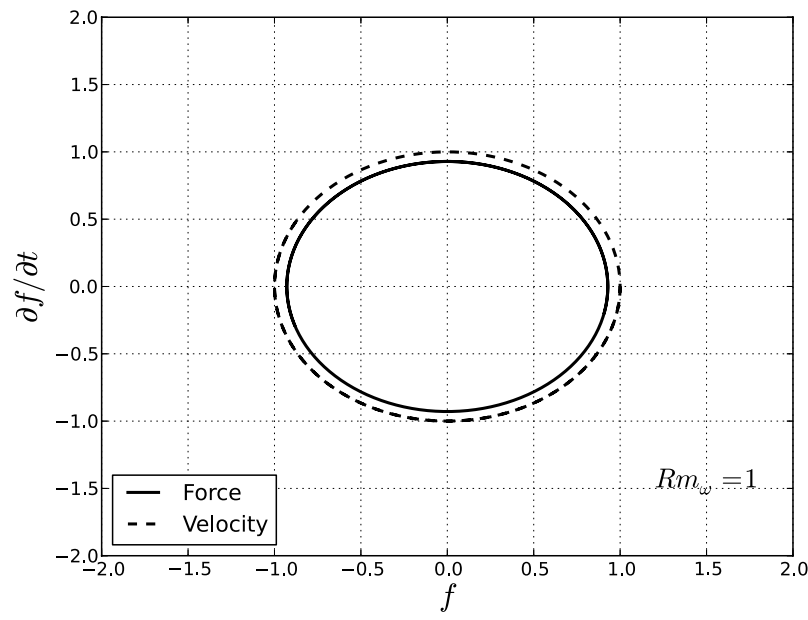

(a)

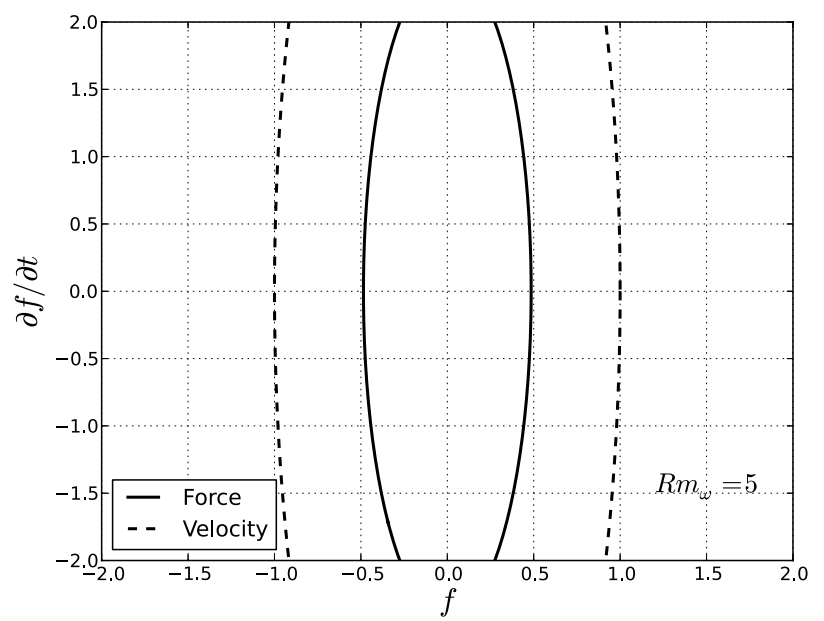

(b)

Fig. 8. (a) Elliptic phase trajectories of $V(t)$ and $\xi(t)$ when $R e_{\omega}=1$ The ellipses are very close to each other which denotes the small phase shift between the functions. (b) When $R e_{\omega} \geq 5$ both ellipses deform significantly. An amplitude of the major axis of $V$-ellipse remains constant, whereas it diminishes in case of $\xi$-ellipse owing to the skin effect.

quickly to a velocity change. The case with infinitely high $R m_{\omega}$ has to be explained in more detail. To understand why the time shift dependency approaches a constant value, it is helpful to consider a phase space. Phase trajectories of velocity and force functions are ellipses, whose geometrical characteristics strongly depend on $R m_{\omega}$. The $V(t)$ and $\xi(t)$ phase curves at $R m_{\omega}=1$ are shown on Fig. 8(a). As ellipsis are close to each other, there is no phase shift between two functions as was mentioned above. But the shape of the ellipses is significantly deformed at $R m_{\omega} \geq 3$ [Fig. 8(b)]. The major axis of the $\xi$-ellipse becomes smaller because of the skin effect, whereas the amplitude of a minor axis tends to infinity as well as the minor axis of $V$-ellipse. At the same time, the major axis of $V$-ellipse remains constant. This tendency results in a fact, that wherever points on the ellipses have been before deformation, the phase angle will be $\pi / 2$. Also this fact has to do with the following physical interpretation: even under high oscillation frequency there are thin skin layers at both sides of the plate where an
TABLE II

REACTION TIME FOR SEVERAL $R m$ OF THE ERROR FUNCTION IN (33)

\begin{tabular}{ccccc}
\hline$R m_{\tau}$ & $T_{98 v}$ & $T_{98 \xi}$ & $T_{98 \psi}$ & $T_{98 v} / T_{98 \xi}$ \\
\hline 1 & 1.65 & 2.36 & 2.64 & 0.70 \\
2 & 0.82 & 1.83 & 2.10 & 0.45 \\
3 & 0.55 & 1.70 & 1.98 & 0.32 \\
4 & 0.41 & 1.64 & 1.92 & 0.25 \\
5 & 0.33 & 1.61 & 1.88 & 0.20 \\
$\infty$ & 0 & 1.48 & 1.76 & 0 \\
\hline
\end{tabular}

equilibrium between advection and diffusion is achieved. If $R m_{\omega} \rightarrow \infty$ the skin layer thickness tends to be zero while increasing the distance between the layers, which leads to their mutual insensitivity and plays a key role in a phase shift phenomenon.

\section{NUMERICAL SOLUTION}

To investigate the time-dependent Lorentz force and Joule heat for arbitrary velocity signals $V(t)$ we use a simple secondorder finite difference code. This code is state of the art and needs no further verification [22], [25]

$$
\begin{aligned}
\frac{b_{i}^{k+1}-b_{i}^{k}}{\Delta t} & =\lambda \frac{b_{i+1}^{k}-2 b_{i}^{k}+b_{i-1}^{k}}{\Delta z^{2}} \\
b_{i}^{k+1} & =(1-2 \gamma) b_{i}^{k}+b_{i-1}^{k}+\gamma b_{i+1}^{k} \\
\gamma & =\lambda \frac{\Delta t}{\Delta z^{2}} .
\end{aligned}
$$

The explicit method has been selected because it is less numerically intensive in comparison with other finite difference methods such as the implicit method or the Crank-Nicolson method. In addition, it is numerically stable and convergent whenever $\gamma \leq 1 / 2$ [22].

The excitation in the form of an error function represents a realistic case of accelerated motion, for instance, a linear drive with motion controller. The error-function is in the form

$$
V(t)=\operatorname{erf}\left[R m_{\tau} \cdot t\right]
$$

If $R m_{\tau}=\infty$ the error function shows identical characteristic as the step function. For small values of $R m_{\tau}$, the error-function has a smaller slope and the Lorentz force and the Joule are almost able to follow the input signal without delay (Table II).

Fig. 9 shows a simple example of a real production process including positive and negative changes in the velocity signal $V(t)$. It can be seen that the Lorentz force can be also positive or negative depending on the moving direction of the plate. In contrast, the Joule heat is always positive and does not depend on the moving direction of the plate. For the considered case of a $0.08-\mathrm{m}$-thick aluminum plate with an electrical conductivity of $\sigma=36 \cdot 10^{6} \mathrm{~S} / \mathrm{m}$ one can obtain the dimensional reaction time of the Lorentz force and Joule heat. To give the reader a feeling of real physical values of $T_{98}$ for the Joule heat and Lorentz force we provide the table in Appendix A. Also one remarkable fact must be mentioned here. As shown in Fig. 9, $T_{98}$ for the Lorentz force is identical for both velocity changes, from $0 \%$ to $100 \%$ and back from $100 \%$ to $0 \%$. But 


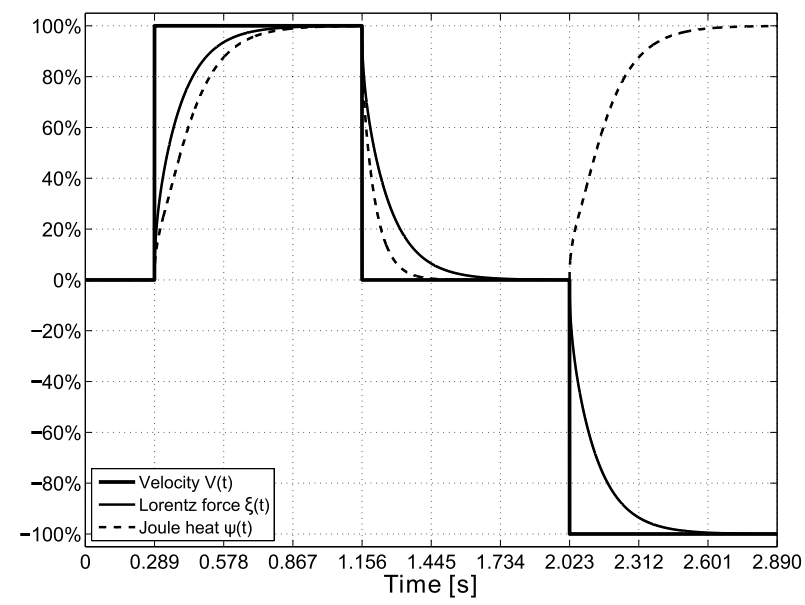

Fig. 9. Time response of Lorentz force and Joule heat for an arbitrary velocity signal $V(t)$. The calculations are performed for an aluminum sheet with an electrical conductivity of $36 \mathrm{MS} / \mathrm{m}$ and a total thickness of $80 \mathrm{~mm}$. The maximum velocity is $1 \mathrm{~m} / \mathrm{s}$ and the primary magnetic field $B_{0}$ has a magnitude of $0.3 T$.

it is not the case for the Joule heat, whose $T_{98}$ is smaller when velocity drops down than when it rises up. In other words, the Joule heat reaches its maximum more slowly than it comes back to zero. Unfortunately, we can explain only the mathematical background of this phenomenon, leaving a physical interpretation unclear. Analytically one can see that the boundary condition for the case of rising velocity is not the same as the condition for the inverse transition. Recalling the analogy with thermal diffusion, the first case implies heating a plate from one side and cooling it down from the another one, whereas in the second case we start with already stationary temperature distribution and let the system evolve in time, while keeping the zero heat flux at the boundaries. This difference in boundary conditions explains inequality of values $T_{98}$ for the Joule heat. On the other hand, one can verify that temperature at the boundaries obeys the same equation in both cases, that is, whatever the direction of velocity change is, the value at the boundaries will evolve equally. As the Lorentz force $\xi(t)$ is basically a value of magnetic field at the boundary, it has the same $T_{98}$ for both transitions.

\section{CONCLUSION}

This paper investigates the interaction of an initially homogenous magnetic field with a moving metal sheet. Both analytical and numerical calculations regarding the timedependent Lorentz force and Joule heat have been performed. To realize a simple model some assumptions have been made. This implies that the plate is infinitely large in $x$ - and $y$-direction and fringing is neglected. The analytical solution has been obtained for three motion setups of the electrically conducting plate. It turns out that both Lorentz force and Joule heat strongly depend on time during transient phase, which causes a remarkable delay compared with the velocity of the plate. In the case of stepwise velocity signal the nondimensional reaction time of the Lorentz force $T_{98 \xi}=1.48$ is smaller than the reaction time of the Joule heat $T_{98 \psi}=1.76$. Different velocity signals cause different shapes of the time
TABLE III

Reference Parameters Which Are Used in the Present Paper

\begin{tabular}{ccc}
\hline Physical value & Reference value & Description \\
\hline$V, m / s$ & $V_{0}$ & Plate velocity \\
$z, m$ & $D$ & Plate thickness \\
$t, s$ & $\mu \sigma D^{2}$ & Diffusion time \\
$b, T$ & $R_{m} B_{0}$ & Induced magnetic field \\
$F, N / m^{2}$ & $\sigma D V_{0} B_{0}^{2}$ & Lorentz force per unit area \\
$Q, W / m^{2}$ & $\sigma^{2} D V_{0}^{2} B_{0}^{2}$ & Joule heat per unit area \\
\hline
\end{tabular}

\section{TABLE IV}

Reaction Time $T_{98}$ in Millisecond Calculated From Fig. 9 For $\mathrm{CU}(\sigma=59 \mathrm{MS} / \mathrm{M})$, AL $(\sigma=36 \mathrm{MS} / \mathrm{m})$ AND STEEL $(\sigma=14 \mathrm{MS} / \mathrm{m})$ FOR DifFERENT Cylinder Diameters. A Time Step WAS $1 \mathrm{~s}$ AND INitial Magnetic Field $B_{0}=0.3 \mathrm{~T}$. A Value Before Slash Corresponds

TO THE LORENTZ ForCe, THE ONE AfTER - TO THE JOUle HeAT

\begin{tabular}{cccccc}
\hline & $\mathrm{D}=20 \mathrm{~mm}$ & $\mathrm{D}=40 \mathrm{~mm}$ & $\mathrm{D}=60 \mathrm{~mm}$ & $\mathrm{D}=80 \mathrm{~mm}$ & $\mathrm{D}=100 \mathrm{~mm}$ \\
\cline { 2 - 6 } $\mathrm{Cu}$ & $44 / 52$ & $176 / 209$ & $395 / 470$ & $702 / 835$ & $1097 / 1305$ \\
$\mathrm{Al}$ & $27 / 32$ & $107 / 127$ & $241 / 287$ & $429 / 510$ & $670 / 796$ \\
$\mathrm{Steel}$ & $10 / 12$ & $42 / 50$ & $94 / 111$ & $167 / 198$ & $260 / 310$ \\
\hline
\end{tabular}

response function. The case of an oscillating motion of the plate is remarkable. First, the Lorentz force shows a drop in the amplitude. Second, a phase shift approaches a constant value at $R m_{\omega} \rightarrow \infty$. Both functions depend on the plate oscillation frequency. This fact is strongly related to skin layers. The Joule heat doubles the frequency, because of the quadratic dependence on the eddy current density.

\section{APPENDIX A}

\section{Nondimensionalized PARAMETERS AND REACTION TIME $T_{98}$ FOR LORENTZ FORCE AND JOULE HEAT}

See Tables III and IV.

\section{APPENDIX B}

\section{DERIVATION OF THE BOUNDARY CONDITION FOR $\partial b / \partial z$}

Here we derive the matching boundary condition for the spatial derivative of $b(z, t)$. The first step is to write down the magnetic field transport equation (1) and to recall the vectors of velocity (2) and magnetic field (3)

$$
\begin{aligned}
\frac{\partial \vec{B}}{\partial t} & =\nabla \times(\vec{V} \times \vec{B})+\lambda \nabla^{2} \vec{B} \\
\vec{V} & =V(t) \vec{e}_{x} \\
\vec{B} & =B_{0} \vec{e}_{z}+b(z, t) \vec{e}_{x} .
\end{aligned}
$$

Then using the identity

$$
\nabla \times(\vec{V} \times \vec{B})=(\vec{B} \nabla) \vec{V}-(\vec{V} \nabla) \vec{B}+\vec{V}(\nabla \vec{B})-\vec{B}(\nabla \vec{V})
$$

we obtain

$$
\nabla \times(\vec{V} \times \vec{B})=B_{0} \frac{\partial \vec{V}}{\partial z}
$$


Therefore, one can verify that

$$
\frac{\partial \vec{b}(z, t)}{\partial t}=B_{0} \frac{\partial \vec{V}}{\partial z}+\lambda \frac{\partial^{2} \vec{b}(z, t)}{\partial z^{2}} .
$$

The next step is to integrate equation (36) over an $\epsilon$-neighborhood of the boundary

$$
\int_{D-\epsilon}^{D+\epsilon} \frac{\partial \vec{b}(z, t)}{\partial t} d z=\int_{D-\epsilon}^{D+\epsilon}\left(B_{0} \frac{\partial \vec{V}}{\partial z}+\lambda \frac{\partial^{2} \vec{b}(z, t)}{\partial z^{2}}\right) d z
$$

Here two facts are important. First, the magnetic field and its time-derivative are assumed to be finite and therefore the integral tends to zero if $\epsilon$ is small enough. Second, the velocity field is nonzero only inside the domain, that is

$$
\vec{V}(z)=\left\{\begin{array}{cc}
\vec{V}(t), & \text { for } z \leq D \\
0, & \text { for } z>D
\end{array}\right.
$$

These arguments simplify the integration and immediately bring us to the boundary condition

$$
\left.\frac{\partial b(z, t)}{\partial z}\right|_{z=D-\epsilon}=-V(t) \frac{B_{0}}{\lambda} .
$$

\section{ACKNOWLEDGMENT}

This work was supported in part by the Deutsche Forschungsgemeinschaft through the framework of the Research Training Group Lorentz force velocimetry and Lorentz force eddy current testing and in part by the BMBF through the framework of the FORMAT-Project.

\section{REFERENCES}

[1] D. Lathrop and C. Forest, "Magnetic dynamos in the lab," Phys. Today, vol. 64 , no. 7 , pp. $40-45,2011$.

[2] N. Weiss, "The expulsion of magnetic flux by eddies," Proc. R. Soc. A, Math. Phys., vol. 293, no. 1434, pp. 310-328, 1966.

[3] P. A. Davidson, An Introduction to Magnetohydrodynamics, 1st ed. Cambridge, U.K.: Cambridge Univ., 2001, p. 452.

[4] H. K. Moffatt, Magnetic Field Generation in Electrically Conducting Fluids, 1st ed. Cambridge, U.K.: Cambridge Univ., 1978, p. 337.

[5] P. Roberts, An Introduction to Magnetohydrodynamics, 1st ed. Cambridge, U.K.: Cambridge Univ., 1967, p. 264
[6] R. Moreau, Magnetohydrodynamics, 1st ed. Dordrecht, The Netherlands: Kluwer Academic, 1990, p. 265.

[7] J. A. Shercliff, A Textbook of Magnetohydrodynamics, 1st ed. New York, NY, USA: Pergamon, 1965, p. 265.

[8] A. Thess, E. Votyakov, B. Knaepen, and O. Zikanov, "Theory of the lorentz force flowmeter," New J. Phys., vol. 9, no. 8, p. 299, Aug. 2007.

[9] A. Thess, E. Votyakov, and Y. Kolesnikov, "Lorentz force velocimetry," Phys. Rev. Lett., vol. 96, no. 16, p. 164501, Apr. 2006.

[10] V. Rudnev, D. Loveless, R. Cook, and M. Black, Handbook of Induction Heating, 1st ed. New York, NY, USA: Marcel Dekker, 2003.

[11] F. Mach, P. Karban, and I. Doleel, "Induction heating of cylindrical nonmagnetic ingots by rotation in static magnetic field generated by permanent magnets," J. Comput. Appl. Math., vol. 236, no. 18, pp. 4732-4744, 2012.

[12] M. Fabbri, M. Forzan, S. Lupi, A. Morandi, and P. Ribani, "Experimental and numerical analysis of DC induction heating of aluminum billets," IEEE Trans. Magn., vol. 45, no. 1, pp. 192-200, Jan. 2009.

[13] R. Parker, "Reconnexion of lines of force in rotating spheres and cylinders," Proc. R. Soc. London A, vol. 291, no. 1424, pp. 60-72, 1966.

[14] M. Perry and T. Jones, "Eddy current induction in a solid conducting cylinder with a transverse magnetic field," IEEE Trans. Magn., vol. 14 no. 4, pp. 227-232, Jul. 1978.

[15] A. Wegfrass et al., "A universal noncontact flowmeter for liquids," Appl. Phys. Lett., vol. 100, no. 19, p. 194103, 2012.

[16] Y. Kolesnikov, C. Karcher, and A. Thess, "Lorentz force flowmeter for liquid aluminum: Laboratory experiments and plant tests," Metall. Mater. Trans. B, vol. 42, no. 3, pp. 441-450, 2011.

[17] C. Weidermann, A. Thess, D. Lieftucht, and M. Reifferscheid, "Application of lorentz force velocimetry in continuous casting of steel," in Proc. 5th Int. Congr. Sci. Technol. Steelmaking, 2012, pp. 1-6.

[18] O. Buneman, The Bennett Pinch, Plasma Physics, J. E. Drummond, Ed. New York, NY, USA: McGraw-Hill, 1961.

[19] S. Molokov, R. Moreau, and H. K. Moffat, Magnetohydrodynamics Historical Evolution and Trends. New York, NY, USA: Springer-Verlag, 2007.

[20] H. H. Woodson and J. R. Melcher, Electromechanical Dynamics, 1st ed. New York, NY, USA: Wiley, 1968, p. 414.

[21] C. Weidermann, "Design and laboratory test of a lorentz force flowmeter for pipe flows," Ph.D. dissertation, Ingenieurwissenschaften und Maschinenbau, Ilmenau Univ. Technol., Ilmenau, Germany, 2013.

[22] J. Crank, The Mathematics of Diffusion, 2nd ed. Oxford, U.K.: Oxford Univ., 1975, p. 414.

[23] H. S. Carslaw and J. C. Jaeger, Conduction of Heat in Solids, 2nd ed. Oxford, U.K.: Clarendon Press, 1959.

[24] F. John, Partial Differential Equations, 1st ed. New York, NY, USA: Springer-Verlag, 1982.

[25] J. W. Thomas, Numerical Partial Differential Equations: Finite Difference Methods, 2nd ed. New York, NY, USA: Springer-Verlag, 1998, p. 460. 